\title{
Utilitaria coreana
}

\section{Korean runabout}

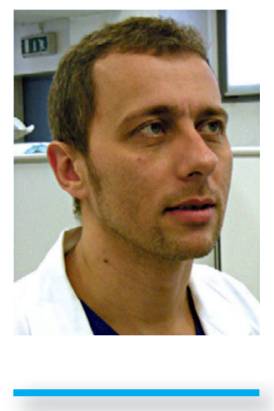

\section{Giovanni Lodi}

Ricercatore

Università degli Studi di Milano UCL Eastman Dental Institute

giovanni.lodi@unimi.it
"Non ho soldi per andare dal dentista".

Sentire qualcuno pronunciare questa frase dovrebbe essere motivo di preoccupazione per ognuno di noi e per la categoria nel suo insieme. Per molto tempo però non è stato così, probabilmente perché la quota di cittadini che invece poteva permettersi le nostre cure era sufficiente a mantenere in salute tutta la filiera.

Adesso le cose sono cambiate. Molte poltrone rimangono vuote per troppo tempo, e ai tanti cittadini il cui salario (quando c'è) è al di sotto della soglia dell'odontoiatria vengono proposti pacchetti "tutto-compreso" da colleghi oltreconfine in grado di richiedere prezzi sensibilmente inferiori a quelli italiani, mentre centri odontoiatrici che offrono troppo a troppo poco invadono i pannelli pubblicitari dei vagoni della metropolitana, spazi un tempo riservati a trattamenti per malattie imbarazzanti o a corsi di recupero per studenti pluriripetenti con genitori facoltosi.

E intanto i termini più cercati in rete comprendono "dentisti croazia", "dentisti convenzionati", "dentisti low-cost” (fatevi un giro su Google Trends, è uno strumento istruttivo).

Ma mi chiedo, e vi chiedo: è possibile una vera odontoiatria low-cost? Non fraintendetemi, non parlo dell'odontoiatria che risparmia su materiali e strumenti, comprimendo i tempi e sottopagando il personale, con tutto ciò che ne consegue. Mi riferisco a un'odontoiatria altra, che trovi soluzioni più semplici e ugualmente efficaci ai problemi dei pazienti. Che riscopra trattamenti ingiustamente accantonati e soprattutto sia capace di inventarne di nuovi. Che permetta di ampliare l'accesso alle cure. Un'odontoiatria che somigli a un'utilitaria coreana. Perfettamente funzionante, garantita, sicura, economica. In grado di soddisfare pienamente un cliente che non si può permettere la Bugatti, e neanche la Golf.

È possibile, secondo Jørgen Slot: nel suo articolo "Low-cost periodontal therapy" pubblicato su Periodontology 2000 Slot illustra una pratica che definisce "efficace, altamente sicura, minimamente invasiva, comoda e poco costosa”. Quasi troppo bello per essere vero. Ma forse non è neppure tanto importante che lo sia. Ciò che importa è che si inizi a pensarci. Perché poi - come diceva qualche giorno fa un mio anziano paziente - se siamo stati capaci di mandare un robot su Marte, vuoi che sia un problema inventare una buona dentiera che costi poco?

Buona lettura. 